\title{
Chaotic behaviors of stable second-order digital filters with two's complement arithmetic
}

\author{
Bingo Wing-Kuen Ling, Wai-Fung Hung and Peter Kwong-Shun Tam \\ Department of Electronic and Information Engineering, The Hong Kong Polytechnic University, Hung \\ Hom, Kowloon, Hong Kong, China
}

\begin{abstract}
SUMMARY
In this paper, the behaviors of stable second-order digital filters with two's complement arithmetic are investigated. It is found that even though the poles are inside the unit circle and the trajectory converges to a fixed point on the phase plane, that fixed point is not necessarily the origin. That fixed point is found and the set of initial conditions corresponding to such trajectories is determined. This set of initial conditions is a set of polygons inside the unit square, whereas it is an ellipse for the marginally stable case. Also, it is found that the occurrence of limit cycles and chaotic fractal pattern on the phase plane can be characterized by the periodic and aperiodic behaviors of the symbolic sequences, respectively. The fractal pattern is polygonal, whereas it is elliptical for the marginally stable case.
\end{abstract}

KEY WORDS: stable; two's complement arithmetic; fractal pattern; limit cycles

\section{INTRODUCTION}

It is well known that chaotic behaviors may occur if second-order digital filters with two's complement arithmetic are operating in the marginally stable region or in the unstable region [1,4,6-10,12,13]. Further results have been reported for marginally stable third-order digital filters with two's complement arithmetic $[3,13]$, as well as for marginally stable second-order digital filters with either a saturationtype nonlinearity [2] or with a quantization-type nonlinearity $[5,11]$.

However, we seldom design a 'digital filter' operating in the marginally stable region or in the unstable region. Practically, we usually design digital filters operating in the stable region. In this paper, chaotic and related behaviors of stable second-order digital filters with two's complement arithmetic are investigated. 
If overflow does not occur, the trajectory of stable second-order digital filters with two's complement arithmetic will converge to the origin on the phase plane. If overflow occurs, will the trajectory converge? If so, what are the fixed points and the set of initial conditions such that the trajectories will converge to those fixed points? Besides, some researchers have reported that limit cycles may occur [14-19]. What is the relationship between the occurrence of limiting cycles and the behaviors of symbolic sequences? Moreover, are there any fractal patterns exhibited on the phase plane? If so, what is the difference between the shapes of fractal patterns for the stable and the marginally stable second-order digital filters?

In section 2, we will outline the notations which are essentially those used in the existing literatures [1-13]. In section 3, the behaviors of stable second-order digital filters with two's complement arithmetic are presented. Comparisons between the behaviors of stable and marginally stable second-order digital filters with two's complement arithmetic are discussed in section 4. Finally, concluding remarks are presented in section 5 .

\section{NOTATIONS}

The notations used in [1-13] are adopted as follows:

The system is defined as:

$\left[\begin{array}{l}x_{1}(k+1) \\ x_{2}(k+1)\end{array}\right]=\mathrm{F}\left(\left[\begin{array}{l}x_{1}(k) \\ x_{2}(k)\end{array}\right]\right)=\left[\begin{array}{c}x_{2}(k) \\ f\left(b \cdot x_{1}(k)+a \cdot x_{2}(k)\right)\end{array}\right]=\mathbf{A} \cdot\left[\begin{array}{l}x_{1}(k) \\ x_{2}(k)\end{array}\right]+\mathbf{B} \cdot s(k)$

where $\mathbf{x}(k)=\left[\begin{array}{l}x_{1}(k) \\ x_{2}(k)\end{array}\right] \in I^{2} \equiv\left\{\left(x_{1}, x_{2}\right):-1 \leq x_{1}<1,-1 \leq x_{2}<1\right\}$

$$
\begin{aligned}
& \mathbf{A}=\left[\begin{array}{ll}
0 & 1 \\
b & a
\end{array}\right] \\
& \mathbf{B}=\left[\begin{array}{l}
0 \\
2
\end{array}\right]
\end{aligned}
$$

$s(k) \in\{-m, \cdots,-1,0,1, \cdots, m\}$ where $m \in Z^{+} \bigcup\{0\}$ satisfying

$-2 \cdot m-1 \leq b \cdot x_{1}+a \cdot x_{2}<2 \cdot m+1$

and $f(x)=x-2 \cdot n$ for $2 \cdot n-1 \leq x<2 \cdot n+1$ and $n \in Z^{+} \bigcup\{0\}$

For the linearized model of the system, if $(a, b) \in \Delta \equiv\{(a, b): b>-1$ and $b<-a+1$ and $b<a+1\}$, the second-order digital filters are said to be operating in the 
stable region. If $b=-1$ and $|a|<2$, or $b=a+1$ and $-2<a<0$, or $b=-a+1$ and $0<a<2$, the second-order digital filters are said to be operating in the marginally stable region. Otherwise, the second-order digital filters are said to be operating in the unstable region.

Given an initial condition $\mathbf{x}(0) \in I^{2}$, a symbolic sequence $s=(s(0), s(1), \cdots) \in \sum$ can be generated by the map $\mathrm{S}: I^{2} \rightarrow \sum$. The set $\sum$ can be partitioned into three subsets: $\Sigma_{\alpha}=\{s=(s(0), s(1), \cdots): s$ is periodic $\}$, $\Sigma_{\beta}=\{s=(s(0), s(1), \cdots): s$ is eventually periodic $\}$ and $\Sigma_{\gamma}=\Sigma \backslash\left(\Sigma_{\alpha} \cup \Sigma_{\beta}\right)$.

\section{BEHAVIORS OF STABLE SECOND-ORDER DIGITAL FILTRS WITH TWO'S COMPLEMENT ARITHMETIC}

This section presents the behaviors of stable second-order digital filters with two's complement arithmetic as follows:

\subsection{Trajectory converging to the origin}

In this section, the trajectory equation and the set of initial conditions for the trajectory to converge to the origin are discussed as below:

Consider the case when the eigenvalues of matrix $\mathbf{A}$ are complex; let $b=-r^{2}$ and $a=2 \cdot r \cdot \cos \theta$

where $r \in \mathfrak{R}^{+}$and $\theta \in \mathfrak{R}$

If $0<r<1$, then the second-order digital filters are stable.

$$
\begin{aligned}
& \text { Let } \mathbf{D}=\left[\begin{array}{cc}
r \cdot e^{j \cdot \theta} & 0 \\
0 & r \cdot e^{-j \cdot \theta}
\end{array}\right] \\
& \mathbf{T}=\left[\begin{array}{cc}
\frac{1}{\sqrt{r}} \cdot e^{-\left(\frac{j \cdot \theta}{2}\right)} & \frac{1}{\sqrt{r}} \cdot e^{\frac{j \cdot \theta}{2}} \\
\sqrt{r} \cdot e^{\frac{j \cdot \theta}{2}} & \sqrt{r} \cdot e^{-\left(\frac{j \cdot \theta}{2}\right)}
\end{array}\right]
\end{aligned}
$$

Then $\mathbf{A}=\mathbf{T} \cdot \mathbf{D} \cdot \mathbf{T}^{-1}$

If overflow does not occur, then

$$
\mathbf{x}(k)=\mathbf{T} \cdot \mathbf{D}^{k} \cdot \mathbf{T}^{-1} \cdot \mathbf{x}(0)=\left[\begin{array}{l}
\frac{r^{k-1}}{\sin \theta} \cdot\left(\sin (k \cdot \theta) \cdot x_{2}(0)-r \cdot \sin ((k-1) \cdot \theta) \cdot x_{1}(0)\right) \\
\frac{r^{k}}{\sin \theta} \cdot\left(\sin ((k+1) \cdot \theta) \cdot x_{2}(0)-r \cdot \sin (k \cdot \theta) \cdot x_{1}(0)\right)
\end{array}\right], \forall k \geq 1
$$


$\Rightarrow \lim _{k \rightarrow+\infty} \mathbf{x}(k)=\mathbf{0}$

Hence, the state vector converges to zero and the phase trajectory converges to the origin. Figure 1a shows an example of the corresponding phase portrait.

$$
\mathbf{x}(k) \in I^{2} \Rightarrow\left|\frac{r^{k}}{\sin \theta} \cdot\left(\sin ((k+1) \cdot \theta) \cdot x_{2}(0)-r \cdot \sin (k \cdot \theta) \cdot x_{1}(0)\right)\right|<1, \forall k \geq 0
$$

If $\sin ((k+1) \cdot \theta)>0$, then

$$
\frac{r \cdot \sin (k \cdot \theta) \cdot x_{1}(0)-\left|\frac{\sin \theta}{r^{k}}\right|}{\sin ((k+1) \cdot \theta)}<x_{2}(0)<\frac{r \cdot \sin (k \cdot \theta) \cdot x_{1}(0)+\left|\frac{\sin \theta}{r^{k}}\right|}{\sin ((k+1) \cdot \theta)}
$$

If $\sin ((k+1) \cdot \theta)<0$, then

$$
\frac{r \cdot \sin (k \cdot \theta) \cdot x_{1}(0)-\left|\frac{\sin \theta}{r^{k}}\right|}{\sin ((k+1) \cdot \theta)}>x_{2}(0)>\frac{r \cdot \sin (k \cdot \theta) \cdot x_{1}(0)+\left|\frac{\sin \theta}{r^{k}}\right|}{\sin ((k+1) \cdot \theta)}
$$

By defining $I_{k}{ }^{2} \equiv\left\{\mathbf{x}(0): \mathbf{x}(0) \in I^{2}\right.$ and $\left.\left|\sin ((k+1) \cdot \theta) \cdot x_{2}(0)-r \cdot \sin (k \cdot \theta) \cdot x_{1}(0)\right|<\left|\frac{\sin \theta}{r^{k}}\right|\right\}$, $\forall k \geq 0$, we can characterize $I_{k}{ }^{2}$ as the region bounded between two parallel straight lines in $I^{2}$ described by the inequalities (15) or (16).

Although the slope of the two parallel straight lines for $I_{k}{ }^{2}$ is different for different values of $k$, the $y$-intercepts of these two lines move away from the origin as $k$ increases. Hence, $\exists K \geq 0$ such that $I_{k}{ }^{2}=I^{2}$ for $k \geq K$. This implies that $\exists K^{\prime} \geq 0$ such that $K^{\prime} \leq K$ and $\bigcap_{k=0}^{+\infty} I_{k}{ }^{2}=\bigcap_{k=0}^{K^{\prime}} I_{k}{ }^{2} \subseteq I^{2}$. As a result, if overflow does not occur, then $\mathbf{x}(0) \in \bigcap_{k=0}^{K^{\prime}} I_{k}{ }^{2}$. This set of initial conditions corresponds to a polygon on the phase plane, and the number of sides of the polygon depends on the value of $K^{\prime}$, as shown in figure $1 b$.

\subsection{Trajectory converging to a fixed point not at the origin}

In this section, the trajectory equation and the set of initial conditions when the trajectory converges to a fixed point not at the origin are developed and discussed as below:

$$
\mathbf{x}(k+1)=\mathbf{A} \cdot \mathbf{x}(k)+\mathbf{B} \cdot s(k), \forall k \geq 0
$$


If $s(k)=s_{0}$ for $\forall k \geq 0$, then

$\mathbf{x}(k)=\mathbf{A}^{k} \cdot \mathbf{x}(0)+\sum_{n=0}^{k-1} \mathbf{A}^{n} \cdot \mathbf{B} \cdot s_{0}$

$=\left[\begin{array}{l}\frac{r^{k-1}}{\sin \theta} \cdot\left(\sin (k \cdot \theta) \cdot x_{2}(0)-r \cdot \sin ((k-1) \cdot \theta) \cdot x_{1}(0)\right) \\ \frac{r^{k}}{\sin \theta} \cdot\left(\sin ((k+1) \cdot \theta) \cdot x_{2}(0)-r \cdot \sin (k \cdot \theta) \cdot x_{1}(0)\right)\end{array}\right]$

$+\frac{2 \cdot s_{0}}{\sin \theta \cdot\left(1-2 \cdot r \cdot \cos \theta+r^{2}\right)} \cdot\left[\begin{array}{l}\sin \theta-r^{k-1} \cdot \sin (k \cdot \theta)+r^{k} \cdot \sin ((k-1) \cdot \theta) \\ \sin \theta-r^{k} \cdot \sin ((k+1) \cdot \theta)+r^{k+1} \cdot \sin (k \cdot \theta)\end{array}\right]$

Since $0<r<1$, we have $\lim _{k \rightarrow+\infty} \mathbf{x}(k)=\frac{2 \cdot s_{0}}{1-2 \cdot r \cdot \cos \theta+r^{2}} \cdot\left[\begin{array}{l}1 \\ 1\end{array}\right]$

Let $\mathbf{x}^{*}=\frac{2 \cdot s_{0}}{1-2 \cdot r \cdot \cos \theta+r^{2}} \cdot\left[\begin{array}{l}1 \\ 1\end{array}\right]$

Then $\mathbf{x}(k)$ will converge to $\mathbf{x}^{*}$. If $s_{0} \neq 0$, then $\mathbf{x}^{*} \neq \mathbf{0}$. Hence, the trajectory converges to a fixed point not at the origin. Figure 2 a shows an example of the corresponding phase portrait. In fact, when $s_{0}=0$, then $\mathbf{x}^{*}=\mathbf{0}$. This reduces to the case described in section 3.1.

$$
\begin{aligned}
& \mathbf{x}(k) \in I^{2} \Rightarrow \mid \frac{r^{k}}{\sin \theta} \cdot\left(\sin ((k+1) \cdot \theta) \cdot x_{2}(0)-r \cdot \sin (k \cdot \theta) \cdot x_{1}(0)\right)+ \\
& \frac{2 \cdot s_{0}}{\sin \theta \cdot\left(1-2 \cdot r \cdot \cos \theta+r^{2}\right)} \cdot\left(\sin \theta-r^{k} \cdot \sin ((k+1) \cdot \theta)+r^{k+1} \cdot \sin (k \cdot \theta)\right) \mid<1 \quad, \forall k \geq 0
\end{aligned}
$$

If $\sin ((k+1) \cdot \theta)>0$, then

$$
\begin{aligned}
& \frac{-\left|\frac{\sin \theta}{r^{k}}\right|-\frac{2 \cdot s_{0}}{1-2 \cdot r \cdot \cos \theta+r^{2}} \cdot\left(\frac{\sin \theta}{r^{k}}-\sin ((k+1) \cdot \theta)+r \cdot \sin (k \cdot \theta)\right)+r \cdot \sin (k \cdot \theta) \cdot x_{1}(0)}{\sin ((k+1) \cdot \theta)} \\
& <x_{2}(0)< \\
& \frac{\left|\frac{\sin \theta}{r^{k}}\right|-\frac{2 \cdot s_{0}}{1-2 \cdot r \cdot \cos \theta+r^{2}} \cdot\left(\frac{\sin \theta}{r^{k}}-\sin ((k+1) \cdot \theta)+r \cdot \sin (k \cdot \theta)\right)+r \cdot \sin (k \cdot \theta) \cdot x_{1}(0)}{\sin ((k+1) \cdot \theta)}
\end{aligned}
$$

If $\sin ((k+1) \cdot \theta)<0$, then 


$$
\begin{aligned}
& \frac{-\left|\frac{\sin \theta}{r^{k}}\right|-\frac{2 \cdot s_{0}}{1-2 \cdot r \cdot \cos \theta+r^{2}} \cdot\left(\frac{\sin \theta}{r^{k}}-\sin ((k+1) \cdot \theta)+r \cdot \sin (k \cdot \theta)\right)+r \cdot \sin (k \cdot \theta) \cdot x_{1}(0)}{\sin ((k+1) \cdot \theta)} \\
& >x_{2}(0)> \\
& \frac{\left|\frac{\sin \theta}{r^{k}}\right|-\frac{2 \cdot s_{0}}{1-2 \cdot r \cdot \cos \theta+r^{2}} \cdot\left(\frac{\sin \theta}{r^{k}}-\sin ((k+1) \cdot \theta)+r \cdot \sin (k \cdot \theta)\right)+r \cdot \sin (k \cdot \theta) \cdot x_{1}(0)}{\sin ((k+1) \cdot \theta)}
\end{aligned}
$$

By defining $I_{k, s_{0}}^{\prime 2} \equiv\left\{\mathbf{x}(0): \mathbf{x}(0) \in I^{2}\right.$ and $\mid \frac{r^{k}}{\sin \theta} \cdot\left(\sin ((k+1) \cdot \theta) \cdot x_{2}(0)-r \cdot \sin (k \cdot \theta) \cdot x_{1}(0)\right)+$ $\left.\frac{2 \cdot s_{0}}{\sin \theta \cdot\left(1-2 \cdot r \cdot \cos \theta+r^{2}\right)} \cdot\left(\sin \theta-r^{k} \cdot \sin ((k+1) \cdot \theta)+r^{k+1} \cdot \sin (k \cdot \theta)\right) \mid<1\right\}, \quad \forall k \geq 0 \quad$ and $\forall s_{0} \neq 0$, we can characterize $I_{k, s_{0}}^{\prime}{ }^{2}$ as the region bounded between two parallel straight lines in $I^{2}$ described by the inequalities (23) or (24). Similarly, $\exists K_{s_{0}}^{\prime} \geq 0$ such that $\bigcap_{k=0}^{+\infty} I_{k, s_{0}}{ }^{2}=\bigcap_{k=0}^{K_{s_{0}}^{\prime}} I_{k, s_{0}}{ }^{2} \subseteq I^{2}$. As a result, if $s(k)=s_{0} \neq 0$ for $\forall k \geq 0$, then $\mathbf{x}(0) \in \bigcup_{\forall s_{0} \neq 0} \bigcap_{k=0}^{K_{s_{0}}^{\prime}} I_{k, s_{0}}^{\prime}{ }^{2}$. This set of initial conditions also corresponds to some polygons on the phase plane, with each polygon associated with a particular value of $s_{0}$ and the number of sides of the polygon depends on the value of $K_{s_{0}}^{\prime}$, as shown in figure $2 \mathrm{~b}$.

\subsection{Occurrence of limit cycles}

In this section, the relationship between the occurrence of limit cycles and the periodic behaviors of the symbolic sequences is developed and discussed as follows:

$$
\forall p, M \in Z^{+} \text {and } \forall k \geq 0, \mathbf{x}(k+p \cdot M)=\mathbf{A}^{p \cdot M} \cdot \mathbf{x}(k)+\sum_{n=0}^{p \cdot M-1} \mathbf{A}^{p \cdot M-1-n} \cdot \mathbf{B} \cdot s(k+n)
$$

If $\exists k_{0} \geq 0$ and $\exists M \in Z^{+}$such that $s(k)=s(k+M)$ for $\forall k \geq k_{0}$, that is $s \in \Sigma_{\alpha} \cup \Sigma_{\beta}$, then $\forall p \in \mathrm{Z}^{+}$

$$
\mathbf{x}\left(k_{0}+p \cdot M\right)=\mathbf{T} \cdot \mathbf{D}^{p \cdot M} \cdot \mathbf{T}^{-1} \cdot \mathbf{x}\left(k_{0}\right)+\sum_{n=0}^{M-1} \mathbf{T} \cdot \mathbf{D}^{M-1-n} \cdot\left(\sum_{m=0}^{p-1} \mathbf{D}^{m \cdot M}\right) \cdot \mathbf{T}^{-1} \cdot \mathbf{B} \cdot s\left(k_{0}+n\right)
$$


$=\mathbf{T} \cdot \mathbf{D}^{p \cdot M} \cdot \mathbf{T}^{-1} \cdot \mathbf{x}\left(k_{0}\right)+$

$\sum_{n=0}^{M-1} \mathbf{T} \cdot\left[\begin{array}{cc}\frac{r^{M-1-n} \cdot e^{j \cdot(M-1-n) \cdot \theta} \cdot\left(1-r^{p \cdot M} \cdot e^{j \cdot p \cdot M \cdot \theta}\right)}{1-r^{M} \cdot e^{j \cdot M \cdot \theta}} & 0 \\ 0 & \frac{r^{M-1-n} \cdot e^{-j \cdot(M-1-n) \theta} \cdot\left(1-r^{p \cdot M} \cdot e^{-j \cdot p \cdot M \cdot \theta}\right)}{1-r^{M} \cdot e^{-j \cdot M \cdot \theta}}\end{array}\right] \cdot \mathbf{T}^{-1} \cdot \mathbf{B} \cdot s\left(k_{0}+n\right)$

$\lim _{p \rightarrow+\infty} \mathbf{x}\left(k_{0}+p \cdot M\right)=\sum_{n=0}^{M-1} \mathbf{T} \cdot\left[\begin{array}{cc}\frac{r^{M-1-n} \cdot e^{j \cdot(M-1-n) \theta}}{1-r^{M} \cdot e^{j \cdot M \cdot \theta}} & 0 \\ 0 & \frac{r^{M-1-n} \cdot e^{-j \cdot(M-1-n) \theta}}{1-r^{M} \cdot e^{-j \cdot M \cdot \theta}}\end{array}\right] \cdot \mathbf{T}^{-1} \cdot \mathbf{B} \cdot s\left(k_{0}+n\right)(29)$

Let $\mathbf{x}_{0}^{*}=\sum_{n=0}^{M-1} \mathbf{T} \cdot\left[\begin{array}{cc}\frac{r^{M-1-n} \cdot e^{j \cdot(M-1-n) \theta}}{1-r^{M} \cdot e^{j \cdot M \cdot \theta}} & 0 \\ 0 & \frac{r^{M-1-n} \cdot e^{-j \cdot(M-1-n) \cdot \theta}}{1-r^{M} \cdot e^{-j \cdot M \cdot \theta}}\end{array}\right] \cdot \mathbf{T}^{-1} \cdot \mathbf{B} \cdot s\left(k_{0}+n\right)$

By defining $\mathbf{x}_{n}{ }^{*}=\mathbf{A}^{n} \cdot \mathbf{x}_{0}{ }^{*}+\sum_{m=0}^{n-1} \mathbf{A}^{n-1-m} \cdot \mathbf{B} \cdot s\left(k_{0}+m\right)$ for $n=1,2, \cdots, M-1$

then $\mathbf{x}(k)$ will converge to a periodic sequence $\left\{\mathbf{x}_{0}{ }^{*}, \mathbf{x}_{1}{ }^{*}, \cdots \mathbf{x}_{M-1}{ }^{*}\right\}$. Hence, limit cycles with period $M$ will occur. Figure $3 \mathrm{a}$ and figure $3 \mathrm{~b}$ shows an example of the corresponding phase portrait and the set of initial conditions that generate limiting cycles, respectively.

\subsection{Chaotic fractal pattern}

In this section, we demonstrate by simulation that when the symbolic sequences are aperiodic, that is, $s \in \Sigma_{\gamma}$, stable second-order digital filters with two's complement arithmetic may also exhibit a chaotic fractal pattern on the phase plane. The fractal pattern is polygonal, whereas it is elliptical for the marginally stable case. Figure $4 \mathrm{a}$ shows an example of the corresponding phase portrait.

Define $\Xi_{0} \equiv\left\{\mathbf{x}(0): s(k)=0, \forall k \geq k_{0}\right\}$. This set is the set of initial conditions that the state trajectories will converge to zero. When $k_{0}=0$, we have found that $\Xi_{0}=\bigcap_{k=0}^{K^{\prime}} I_{k}{ }^{2}$. Similarly, define $\Xi_{1} \equiv\left\{\mathbf{x}(0): s(k)=s_{0} \neq 0, \forall k \geq k_{0}^{1}\right\}$. This set is the set of initial conditions that the state trajectories will converge to a fixed point. When $k_{0}^{1}=0$, we have found that $\Xi_{1}=\bigcup_{\forall s_{0} \neq 0} \bigcap_{k=0}^{K_{s_{0}}^{\prime}} I_{k, s_{0}}^{\prime}{ }^{2}$. Likewise, we define $\Xi_{M} \equiv\left\{\mathbf{x}(0): s(k)=s(k+M)\right.$, for $k \geq k_{0}^{M}$ and for $\left.M>1\right\}$. This set is the set of initial 
conditions that generates limiting cycles. If $\Xi_{3}=I^{2} \backslash \bigcup_{M \geq 0} \Xi_{M} \neq \varnothing$, this implies that there exists some initial conditions which may not result in the convergence of the state trajectories nor the occurrence of limiting cycles. Under this condition, chaotic behaviors may occur.

Since $\forall k \geq 0, \mathbf{x}(k)$ will not fall into the set $\bigcup_{M \geq 0} \Xi_{M}$, otherwise, $\exists k_{0} \geq 0$ such that $\mathbf{x}\left(k_{0}\right) \in \bigcup_{M \geq 0} \Xi_{M}$. As a result, the phase portrait is inside $\Xi_{3}=I^{2} \backslash \bigcup_{M \geq 0} \Xi_{M}$. This may correspond to polygonal fractal patterns because $\Xi_{M} \forall M \geq 0$ are all polygons. Figure $4 \mathrm{~b}$ shows an example of the set of initial conditions that gives polygonal fractal patterns.

\section{COMPARISONS BETWEEN THE BAHAVIORS OF STABLE AND}

\section{MARGINALLY STABLE SECOND-ORDER DIGITAL FILTERS WITH TWO'S COMPLEMENT ARITHMETIC}

Table 1 summarizes the differences on the behaviors of the state trajectories $\mathbf{x}(k)$ between stable and marginally stable second-order digital filters with two's complement arithmetic. Table 2 summarizes the corresponding differences on the phase portrait. Table 3 summarizes the differences on the sets of initial conditions for the occurrence of various symbolic sequences between stable and marginally stable second-order digital filters with two's complement arithmetic.

\begin{tabular}{|c|c|c|c|c|}
\hline & $s(k)=0$, & $s(k)=s_{0} \neq 0$, & $s \in \Sigma_{\alpha} \cup \Sigma_{\beta}$ & $s \in \Sigma_{\gamma}$ \\
& $\forall k \geq 0$ & $\forall k \geq 0$ & & \\
\hline state trajectory & oscillates with & oscillates with & oscillates with & chaotic \\
$\mathbf{x}(k)$ of & its natural & its natural & harmonic & \\
marginally & frequency, and & frequency, and & frequencies & \\
stable second- & the DC value=0 & the DC value $\neq 0$ & $\frac{2 \cdot \pi \cdot n}{M}$, for & \\
order digital & & $n=0,1, \cdots, M-1$ & \\
filters with & & & & \\
two's & & & & \\
complement & & & & \\
arithmetic & & & & \\
\hline
\end{tabular}




\begin{tabular}{|c|c|c|c|c|}
\hline state trajectory & converges to & converges to a & limit cycles & chaotic \\
$\mathbf{x}(k)$ of stable & zero & non-zero value & occur with & \\
second-order & & & period $M$ & \\
digital filters & & & \\
with two's & & & \\
complement & & & \\
arithmetic & & & \\
\hline
\end{tabular}

Table 1. Comparison of the behaviors of state trajectories $\mathbf{x}(k)$ between stable and marginally stable second-order digital filters with two's complement arithmetic, for the initial conditions in Table 3.

\begin{tabular}{|c|c|c|c|c|}
\hline & $\begin{array}{l}s(k)=0, \\
\forall k \geq 0\end{array}$ & $\begin{array}{c}s(k)=s_{0} \neq 0, \\
\forall k \geq 0\end{array}$ & $s \in \Sigma_{\alpha} \cup \Sigma_{\beta}$ & $s \in \Sigma_{\gamma}$ \\
\hline $\begin{array}{c}\text { phase portrait of } \\
\text { marginally } \\
\text { stable second- } \\
\text { order digital } \\
\text { filters with } \\
\text { two's } \\
\text { complement } \\
\text { arithmetic }\end{array}$ & $\begin{array}{l}\text { single ellipse } \\
\text { centered at the } \\
\text { origin }\end{array}$ & $\begin{array}{l}\text { several ellipses } \\
\text { with same size, } \\
\text { and all the } \\
\text { centers are not } \\
\text { at the origin }\end{array}$ & $\begin{array}{c}\text { several ellipses } \\
\text { with different } \\
\text { sizes, and all the } \\
\text { centers are not } \\
\text { at the origin }\end{array}$ & $\begin{array}{c}\text { elliptical fractal } \\
\text { pattern }\end{array}$ \\
\hline $\begin{array}{c}\text { phase portrait of } \\
\text { stable second- } \\
\text { order digital } \\
\text { filters with } \\
\text { two's } \\
\text { complement } \\
\text { arithmetic }\end{array}$ & $\begin{array}{c}\text { converges to the } \\
\text { origin }\end{array}$ & $\begin{array}{l}\text { converges to a } \\
\text { fixed point, and } \\
\text { the fixed point } \\
\text { is not at the } \\
\text { origin }\end{array}$ & $\begin{array}{c}\text { converges to } \\
\text { several fixed } \\
\text { points, and } \\
\text { those fixed } \\
\text { points are not at } \\
\text { the origin }\end{array}$ & $\begin{array}{c}\text { polygonal } \\
\text { fractal pattern }\end{array}$ \\
\hline
\end{tabular}

Table 2. Comparison of the phase portraits between stable and marginally stable second-order digital filters with two's complement arithmetic, for the initial conditions in Table 3. 


\begin{tabular}{|c|c|c|c|c|}
\hline & $\begin{array}{l}s(k)=0, \\
\forall k \geq 0\end{array}$ & $\begin{array}{c}s(k)=s_{0} \neq 0, \\
\forall k \geq 0\end{array}$ & $s \in \Sigma_{\alpha} \cup \Sigma_{\beta}$ & $S \in \Sigma_{\gamma}$ \\
\hline $\begin{array}{l}\text { set of initial } \\
\text { conditions of } \\
\text { marginally } \\
\text { stable second- } \\
\text { order digital } \\
\text { filters with } \\
\text { two's } \\
\text { complement } \\
\text { arithmetic }\end{array}$ & $\begin{array}{l}\text { single ellipse } \\
\text { centered at the } \\
\text { origin }\end{array}$ & $\begin{array}{l}\text { several ellipses } \\
\text { with same size, } \\
\text { and all the } \\
\text { centers are not } \\
\text { at the origin }\end{array}$ & $\begin{array}{c}\text { several ellipses } \\
\text { with different } \\
\text { sizes, and all the } \\
\text { centers are not } \\
\text { at the origin }\end{array}$ & $\begin{array}{c}\text { elliptical fractal } \\
\text { pattern }\end{array}$ \\
\hline $\begin{array}{l}\text { set of initial } \\
\text { conditions of } \\
\text { stable second- } \\
\text { order digital } \\
\text { filters with } \\
\text { two's } \\
\text { complement } \\
\text { arithmetic }\end{array}$ & $\begin{array}{l}\text { single polygon } \\
\text { centered at the } \\
\text { origin }\end{array}$ & $\begin{array}{c}\text { several } \\
\text { polygons with } \\
\text { same size, and } \\
\text { all the centers } \\
\text { are not at the } \\
\text { origin }\end{array}$ & $\begin{array}{l}\text { several } \\
\text { polygons with } \\
\text { different sizes, } \\
\text { and all the } \\
\text { centers are not } \\
\text { at the origin }\end{array}$ & $\begin{array}{c}\text { polygonal } \\
\text { fractal pattern }\end{array}$ \\
\hline
\end{tabular}

Table 3. Comparison of the sets of initial conditions for the occurrence of various symbolic sequences between stable and marginally stable second-order digital filters with two's complement arithmetic.

\section{CONCLUDING REMARKS}

In this paper, we have studied the behaviors of stable second-order digital filters with two's complement arithmetic. It is found that the state vector of stable second-order digital filters with two's complement arithmetic may not converge to zero. The sets of initial conditions that the trajectories converge to those fixed points are polygons inside the unit square. Also, it is found that the occurrence of limit cycles and chaotic fractal patterns on the phase plane can be characterized by the periodic and aperiodic behaviors of the symbolic sequences, respectively. The fractal pattern is polygonal, whereas it is elliptical for the marginally stable case. 


\section{ACKNOWLEDGEMENT}

The work described in this paper was substantially supported by The Hong Kong Polytechnic University.

\section{REFERENCES}

1. Chua LO, Lin T. Chaos in digital filters. IEEE Transactions on Circuits and Systems 1988; 35(6):648-658.

2. Galias Z, Ogorzalek MJ. Bifurcation phenomena in second-order digital filter with saturationtype adder overflow characteristic. IEEE Transactions on Circuits and Systems 1990; 37(8):1068-1070.

3. Chua LO, Lin T. Chaos and fractals from third-order digital filters. International Journal of Circuit Theory and Applications 1990; 18:241-255.

4. Chua LO, Lin T. Fractal pattern of second-order non-linear digital filters: a new symbolic analysis. International Journal of Circuit Theory and Applications 1990; 18:541-550.

5. Lin T, Chua LO. On chaos of digital filters in the real world. IEEE Transactions on Circuits and Systems 1991; 38(5):557-558.

6. Galias Z, Ogorzalek MJ. On symbolic dynamics of a chaotic second-order digital filter. International Journal of Circuit Theory and Applications 1992; 20:401-409.

7. Wu CW, Chua LO. Properties of admissible symbolic sequences in a second-order digital filter with overflow non-linearity. International Journal of Circuit Theory and Applications 1993; 21:299-307.

8. Kocarev L, Chua LO. On chaos in digital filters: case $b=-1$. IEEE Transactions on Circuits and Systems - II: Analog and Digital Signal Processing 1993; 40(6):404-407.

9. Kocarev L, Wu CW, Chua LO. Complex behavior in digital filters with overflow nonlinearity: analytical results. IEEE Transactions on Circuits and Systems - II: Analog and Digital Signal Processing 1996; 43(3):234-246.

10. Yu X, Galias Z. Periodic behaviors in a digital filter with two's complement arithmetic. IEEE Transactions on Circuits and Systems-1: Fundamental Theory and Applications 2001; 48(10):1177-1190.

11. Ling BWK, Luk FCK, Tam PKS. Further investigation on chaos of real digital filters. International Journal of Bifurcation and Chaos 2003; 13(2):493-495.

12. Ling WK, Tam PKS. Some new trajectory patterns and periodic behaviors of unstable second-order digital filter with two's complement arithmetic. International Journal of Bifurcation and Chaos 2003; 13(9).

13. Ling WK, Hung WF, Tam PKS. Chaotic behaviors of a digital filter with two's complement arithmetic and arbitrary initial conditions and order. International Journal of Bifurcation and Chaos 2004; 14(7). 
14. Sandberg IW, Kaiser JF. A bound on limit cycles in fixed-point implementations of digital filters. IEEE Transactions on Audio and Electroacoustics 1972; AU-20(2):110-112.

15. Parker SR, Hess SF. Limit-cycle oscillations in digital filters. IEEE Transactions on Circuit Theory 1971; CT-18(6):687-697.

16. Thông T, Liu B. Limit cycles in the combinatorial implementation of digital filters. IEEE Transactions on Acoustics, Speech, and Signal Processing 1976; ASSP-24(3):248-256.

17. Barnes CW, Fam AT. Minimum norm recursive digital filters that are free of overflow limit cycles. IEEE Transactions on Circuits and Systems 1977; CAS-24(10):569-574.

18. Mills WL, Mullis CT, Roberts RA. Digital filter realizations without overflow oscillations. IEEE Transactions on Acoustics, Speech, and Signal Processing 1978; ASSP-26(4):334-338.

19. Munson DC, Strickland JH, Walker TP. Maximum amplitude zero-input limit cycles in digital filters. IEEE Transactions on Circuits and Systems 1984; CAS-31(3):266-275. 

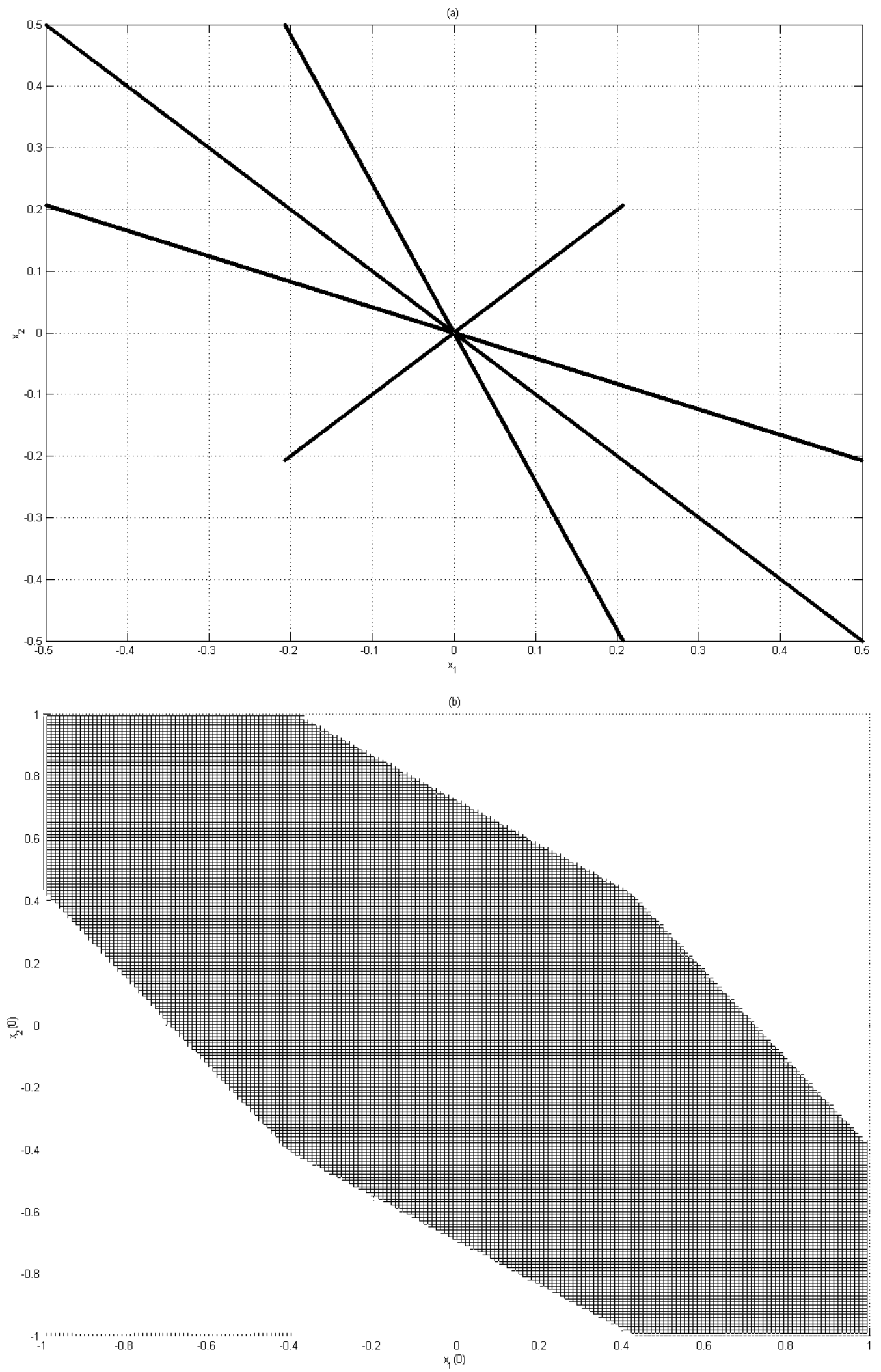

Figure 1. (a) The phase portrait of stable second-order digital filters with two's complement arithmetic, $r=0.9999, \theta=0.75 \cdot \pi$ and $\mathbf{x}(0)=\left[\begin{array}{c}0.5 \\ -0.5\end{array}\right]$. (b) The set of initial conditions of the corresponding digital filters when $s(k)=0 \quad \forall k \geq 0$. 

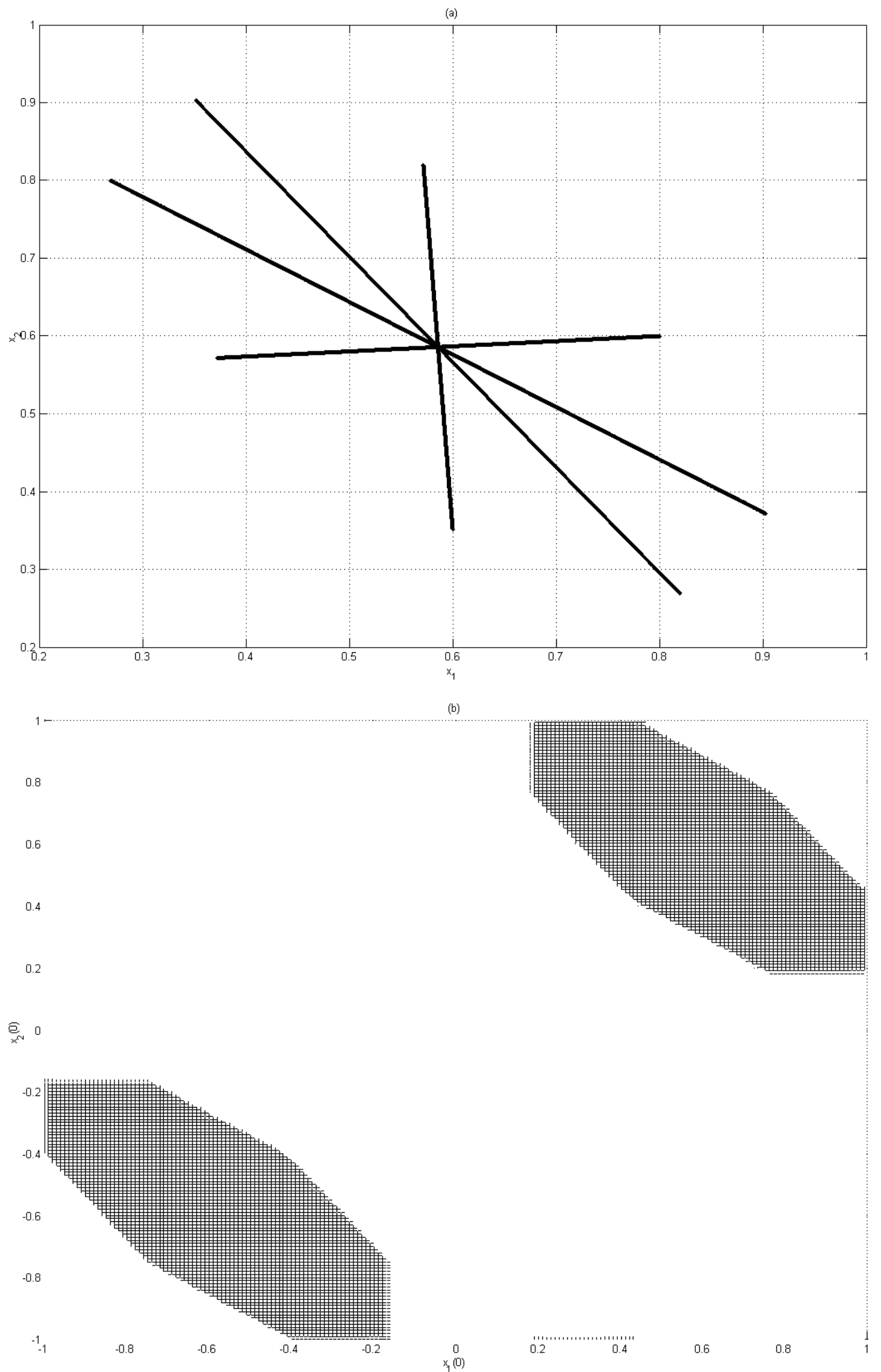

Figure 2. (a) The phase portrait of stable second-order digital filters with two's complement arithmetic, $r=0.9999, \theta=0.75 \cdot \pi$ and $\mathbf{x}(0)=\left[\begin{array}{l}0.8 \\ 0.6\end{array}\right]$. (b) The set of initial conditions of the corresponding digital filters when $s(k)=s_{0} \neq 0 \quad \forall k \geq 0$. 

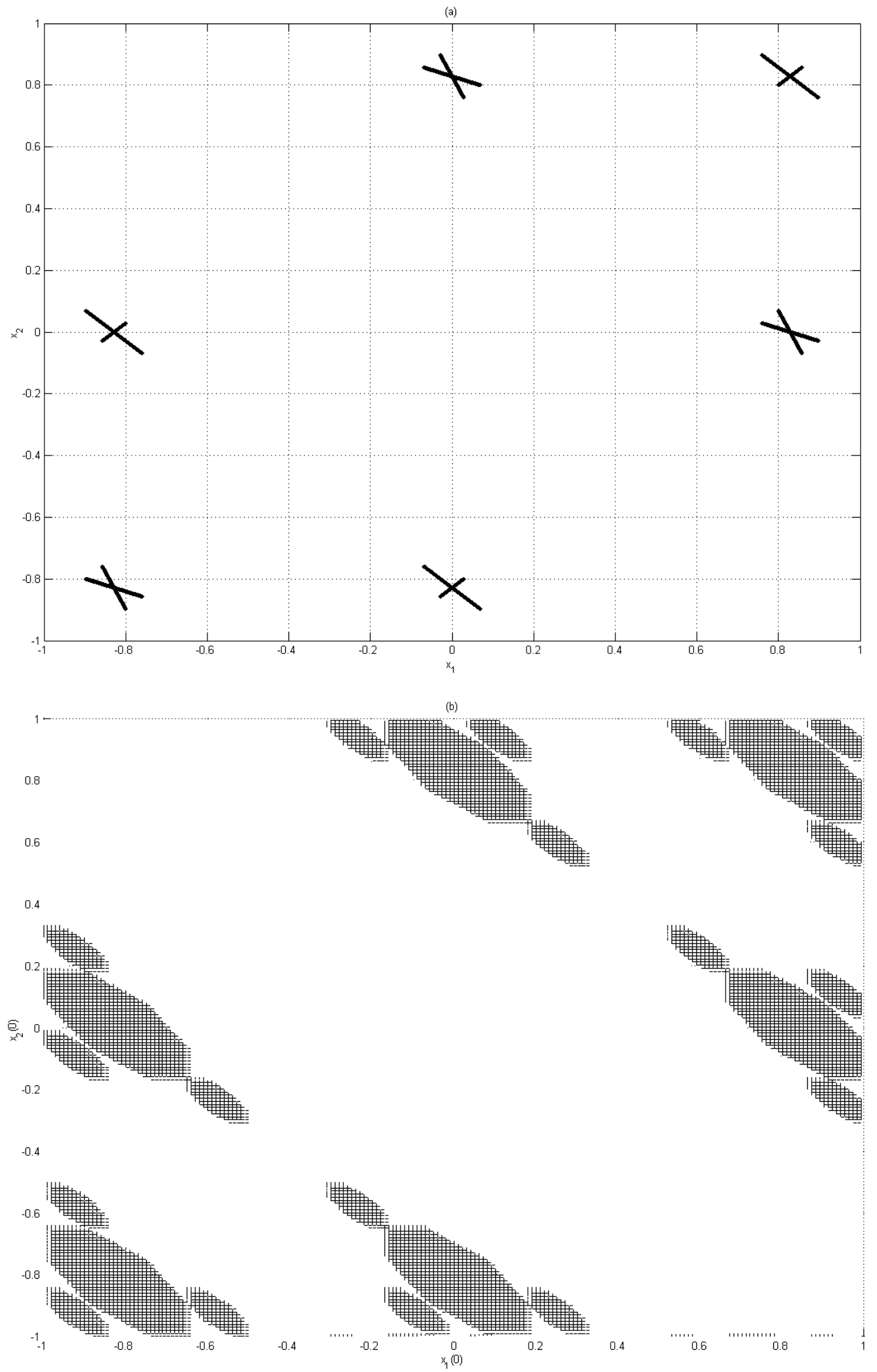

Figure 3. (a) The phase portrait of stable second-order digital filters with two's complement arithmetic, $r=0.9999, \theta=0.75 \cdot \pi$ and $\mathbf{x}(0)=\left[\begin{array}{l}0.8 \\ 0.6\end{array}\right]$. (b) The set of initial conditions of the corresponding digital filters when $s(k)=s(k+M) \forall k \geq 0$. 

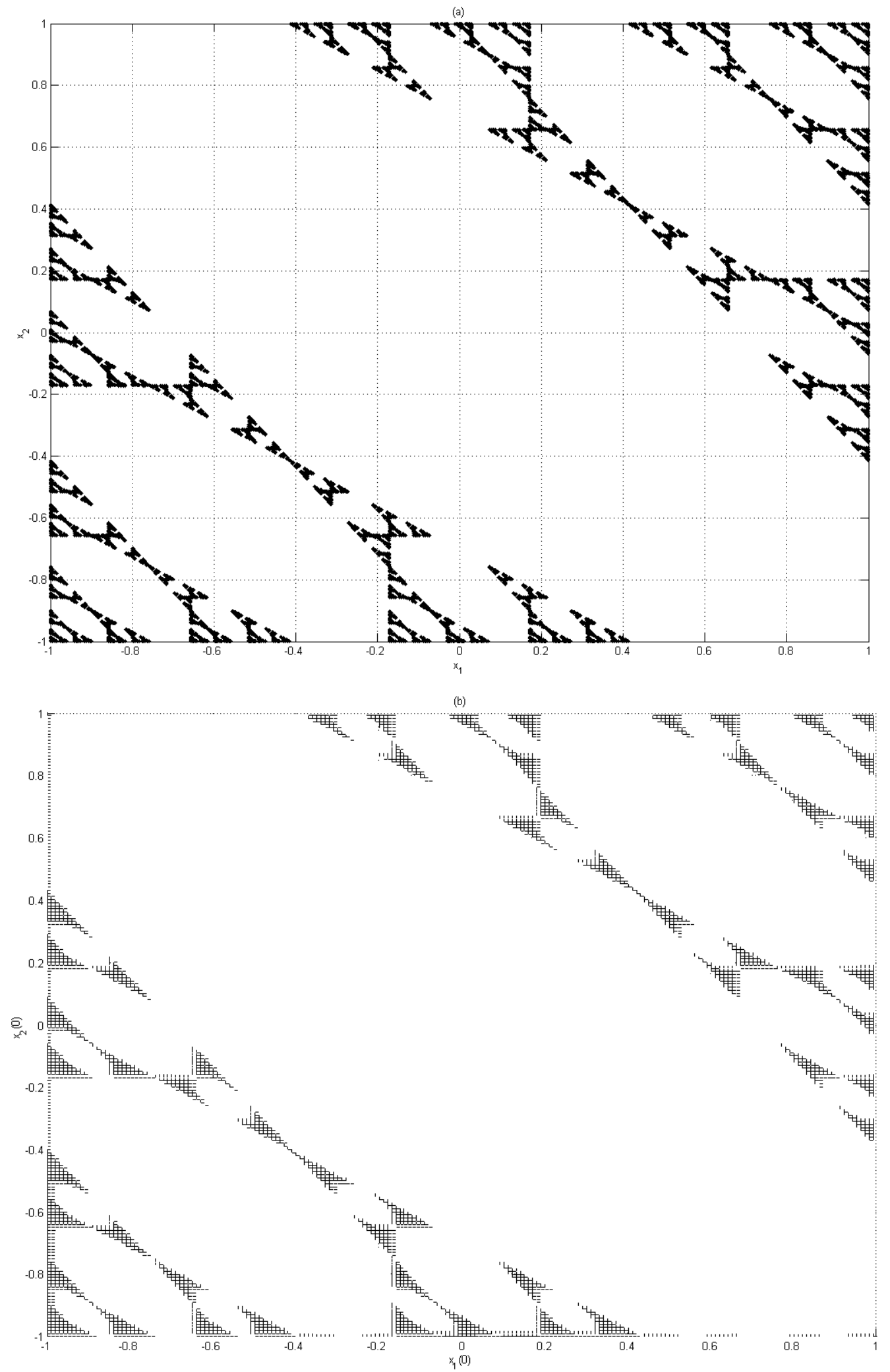

Figure 4. (a) The phase portrait of stable second-order digital filters with two's complement arithmetic, $r=0.9999, \theta=0.75 \cdot \pi$ and $\mathbf{x}(0)=\left[\begin{array}{c}-1 \\ 0\end{array}\right]$. (b) The set of initial conditions of the corresponding digital filters when $s(k)$ is aperiodic. 